\title{
Image quality of late gadolinium enhancement in cardiac magnetic resonance with different doses of contrast material in patients with chronic myocardial infarction
}

\author{
Caterina Beatrice Monti ${ }^{1}$, Marina Codari ${ }^{2}$, Andrea Cozzi ${ }^{1 *}$ (D), Marco Ali ${ }^{3,4}$, Lorenzo Saggiante ${ }^{5}$, \\ Francesco Sardanelli, $i^{3,6}$ and Francesco Secchi $i^{3,6}$
}

\begin{abstract}
Background: Contrast-enhanced cardiac magnetic resonance (CMR) is pivotal for evaluating chronic myocardial infarction (CMI). Concerns about safety of gadolinium-based contrast agents favour dose reduction. We assessed image quality of scar tissue in CMRs performed with different doses of gadobutrol in CMI patients.

Methods: Informed consent was waived for this Ethics Committee-approved single-centre retrospective study. Consecutive contrast-enhanced CMRs from CMI patients were retrospectively analysed according to the administered gadobutrol dose (group A, $0.10 \mathrm{mmol} / \mathrm{kg}$; group B, $0.15 \mathrm{mmol} / \mathrm{kg}$; group C, $0.20 \mathrm{mmol} / \mathrm{kg}$ ). We calculated the signal-tonoise ratio for scar tissue ( $\left(\mathrm{SR}_{\text {scar }}\right)$ and contrast-to-noise ratio between scar and either remote myocardium (CNR $\mathrm{R}_{\text {scar-rem }}$ ) or blood (CNR scar-blood).

Results: Of 79 CMRs from 79 patients, 22 belonged to group A, 26 to group B, and 31 to group C. The groups were homogeneous for age, sex, left ventricular morpho-functional parameters, and percentage of scar tissue over whole myocardium ( $p \geq 0.300)$. SNR scar $_{\text {was }}$ lower in group $A(46.4 ; 40.3-65.1)$ than in group $B(70.1 ; 52.2-111.5)(p=0.013)$ and group $C(72.1 ; 59.4-100.0)(p=0.002)$, $C N R_{\text {scar-rem }}$ was lower in group $A(62.9 ; 52.2-87.4)$ than in group $B$ $(96.5 ; 73.1-152.8)(p=0.008)$ and in group $C(103.9 ; 83.9-132.0)(p=0.001)$. No other significant differences were found $(p \geq 0.335)$.

Conclusions: Gadobutrol at $0.10 \mathrm{mmol} / \mathrm{kg}$ provides inferior scar image quality of CMI than 0.15 and $0.20 \mathrm{mmol} / \mathrm{kg}$; the last two dosages seem to provide similar LGE. Thus, for CMR of CMl, $0.15 \mathrm{mmol} / \mathrm{kg}$ of gadobutrol can be suggested instead of $0.20 \mathrm{mmol} / \mathrm{kg}$, with no hindrance to scar visualisation. Dose reduction would not impact on diagnostic utility of CMR examinations.
\end{abstract}

Keywords: Contrast media, Gadobutrol, Gadolinium, Magnetic resonance imaging, Myocardial infarction

\footnotetext{
* Correspondence: andrea.cozzi1@unimi.it

This article is part of a thematic series on Myocardial tissue characterization

in ischemic heart disease (Guest Editors: Akos Varga-Szemes and Pal Suranyi,

MUSC Health Charleston, SC, United States).

'Department of Biomedical Sciences for Health, Università degli Studi di

Milano, Via Mangiagalli 31, 20133 Milano, Italy

Full list of author information is available at the end of the article
}

\section{Springer Open}

( ) The Author(s). 2020 Open Access This article is licensed under a Creative Commons Attribution 4.0 International License, which permits use, sharing, adaptation, distribution and reproduction in any medium or format, as long as you give appropriate credit to the original author(s) and the source, provide a link to the Creative Commons licence, and indicate if changes were made. The images or other third party material in this article are included in the article's Creative Commons licence, unless indicated otherwise in a credit line to the material. If material is not included in the article's Creative Commons licence and your intended use is not permitted by statutory regulation or exceeds the permitted use, you will need to obtain permission directly from the copyright holder. To view a copy of this licence, visit http://creativecommons.org/licenses/by/4.0/. 


\section{Key points}

- Late gadolinium enhancement is pivotal in assessing chronic myocardial infarction.

- Safety issues of gadolinium-based contrast agents advocate for dose reduction.

- Gadobutrol at $0.10 \mathrm{mmol} / \mathrm{kg}$ showed lower scar quality compared to higher doses.

- Gadobutrol at $0.15 \mathrm{mmol} / \mathrm{kg}$ provides comparable image quality to $0.20 \mathrm{mmol} / \mathrm{kg}$.

- Gadobutrol at $0.15 \mathrm{mmol} / \mathrm{kg}$ can be suggested for assessing chronic myocardial infarction.

\section{Background}

Coronary heart disease is one of the main causes of morbidity and mortality, especially in developed countries, where it causes around 20\% of all deaths [1]. The most common presentation of coronary heart disease is myocardial infarction, which is defined as the occurrence of necrosis in the setting of myocardial ischaemia[2].

Contrast-enhanced cardiac magnetic resonance (CMR) is a multi-parametric, multi-planar imaging technique, which represents the current non-invasive standard of care for assessing cardiac volumes, function and tissue characterisation through late gadolinium enhancement (LGE) [3, 4]. The importance of LGE may be found, among other reasons, in its prognostic potential [5]. Given its capability to monitor cardiac conditions, contrastenhanced CMR may be useful in the evaluation of patients with chronic myocardial infarction, especially when the latter is transmural and of greater clinical relevance $[6,7]$. Moreover, automatic scar quantification is growing in popularity due to the increase in numbers of examinations and the development of increasingly more reliable methods [8]. Scar recognition is most often based on image characteristics of the scarred area, such as signal(SNR) and contrast-to-noise ratio (CNR) [9].

However, especially in the latest years, concerns about the safety of gadolinium-based contrast agents (GBCA) have arisen. In addition to the well-known issue of nephrogenic systemic fibrosis [10], gadolinium deposits of yet unknown clinical relevance have been shown in the brain of patients, adults and children, who underwent repeated GBCA-enhanced magnetic resonance examinations [11, 12]. This led to a growing attention concerning the possibility to reduce GBCA doses in such examinations, provided that scar quality is not hindered.

Patients with chronic myocardial infarction hence represent a population where GBCA dose reduction would lead to a lower chance of contrast-related adverse events. At present, GBCA doses used in these patients are variable among countries and centres, usually between 0.1 (single dose) and 0.2 (double dose) $\mathrm{mmol} / \mathrm{kg}$ [13]. While in some countries, such as Japan, the single dose is recommended, in most cases there are no specific indications [14]. All doses seem to provide diagnostic quality to examinations, albeit a reduction in scar visualisation corresponding to a lower contrast dosage might, for instance, hinder post-processing applications.

The purpose of our study was to analyse image quality of the scar tissue in CMR examinations performed with different GBCA doses in patients with chronic transmural myocardial infarction, to investigate the impact of gadolinium dose variation on the visibility of myocardial LGE quantified as SNR and CNR.

\section{Methods}

\section{Ethical statement and study design}

The local Ethics Committee approved this study (Ethics Committee of IRCCS Ospedale San Raffaele; protocol code "Cardioretro Ricerca Spontanea"; approved on September 14, 2017, and amended on July 18, 2019). This study was supported by local research funds of IRCCS Policlinico San Donato, a clinical research hospital partially funded by the Italian Ministry of Health. This research received no specific grant from funding agencies in the public, commercial, or non-profit sector. Due to the retrospective nature of this study, specific informed consent was waived.

\section{Study population}

All patients who had undergone a contrast-enhanced CMR examination with administration of gadobutrol (Gadovist, Bayer Healthcare, Leverkusen, Germany), at our institution between March 2014 (the introduction of our newer magnetic resonance unit) and May 2018, and who were diagnosed with chronic myocardial infraction from clinical findings and CMR, were included in our study. Exclusion criteria were the presence of oedema, indicating acute phase of infarction, presence of relevant artefacts which rendered differentiation of the myocardial scar difficult, and non-transmural, thin infarcts which were either only subendocardial $(\leq 50 \%$ of wall thickness) or too small (scar $\leq 10 \%$ of the myocardium), as such conditions do not allow the calculation of SNR and $\mathrm{CNR}$ of the scarred region [15]. Moreover, in patients with subendocardial infarction, image contrast may vary according to acquisition timing, and thus this may provide data that are not compatible with those of transmural scars [16].

Patients were then divided into three subgroups, depending on the contrast dose administered during their CMR: the first group (A) received $0.10 \mathrm{mmol} / \mathrm{kg}$, the second (B) $0.15 \mathrm{mmol} / \mathrm{kg}$, and the third (C) $0.20 \mathrm{mmol} /$ $\mathrm{kg}$. These different doses were mainly due to choices of the physicians in charge of the examination during the study period, not related to a specific patient's condition. 


\section{Image acquisition}

All subjects were imaged using one 1.5 - $\mathrm{T}$ whole-body magnetic resonance unit (Magnetom Aera, Siemens Healthineers, Erlangen, Germany) with $45 \mathrm{mT} / \mathrm{m}$ gradient power and an 18-channel surface phased-array coil. The examined patient was lying supine and the coil was placed over the thorax. All images were acquired with breath-holding and ECG gating.

The imaging protocol of all patients included cine and LGE sequences.

Cine images were acquired in multiple short- and long-axis planes using an ECG-triggered bright-blood steady-state free-precession pulse sequence.

LGE images were acquired after intravenous administration of $0.10,0.15$, or $0.20 \mathrm{mmol} / \mathrm{kg}$ of gadobutrol (Gadovist, Bayer Healthcare, Leverkusen, Germany) and were performed using a $2 \mathrm{D}$ segmented inversionrecovery fast gradient-echo sequence covering the entire left ventricle. Earlier exams utilised higher-contrast doses, which were then lowered over time. Nevertheless, the sequence for LGE imaging remained the same. The time of echo was $3.33 \mathrm{~ms}$, while the time of repetition was adapted to patients' heart rates, and inversion time was progressively modified from 260 to $330 \mathrm{~ms}$, to blacken cardiac muscle; flip angle was $25^{\circ}$, slice thickness $8 \mathrm{~mm}$, and pixel size $3.6 \mathrm{~mm}^{2}$. LGE images were reconstructed using magnitude reconstruction. From the $R$ wave of the electrocardiogram, a delay period was used to ensure that image acquisition occurred in middiastole, when the heart is relatively motionless, therefore reducing motion artefacts. Data were acquired every other heartbeat, although in tachycardic patients data were acquired every third heartbeat, while in bradycardic patients and in patients with difficulties in breath holding acquisition was performed every heartbeat. Timing between contrast administration and acquisition of delayed enhancement scans was tailored to the contrast dose that was utilised in each case, according to literature recommendations [14].

\section{Image analysis}

Image analysis was performed using QMass 7.6 (Medis Medical Imaging Systems, Leiden, The Netherlands). The epicardial contour of the left ventricle was manually traced for all short-axis slices at end-diastolic and end-systolic phases in cine sequences. Afterwards, a bloodthresholding technique (Mass-K mode) was applied to automatically segment myocardium and blood pool. The software then calculated end-diastolic and end-systolic volumes, both indexed and non-indexed to body surface area, myocardial mass, stroke volume, and ejection fraction.

For LGE quantification, manual segmentation of endocardium and epicardium of the left ventricle was performed in inversion recovery sequences after contrast agent injection. Then the software automatically detected the myocardial scar as being 6 standard deviations above average myocardial intensity [17]. Manual corrections were made when the software erroneously detected additional scarred areas, or when it failed to properly detect the scar. LGE was quantified as percentage over the whole myocardium. Two regions of interest were automatically placed in the scarred and healthy myocardium. An example of LGE segmentation is shown in Fig. 1.

SNR and CNR were calculated using data provided by automatic LGE quantification, namely intensities from the two ROIs automatically placed in the scarred and healthy myocardium, and two additional ROIs traced in the left ventricular blood pool and in the background air. SNR was calculated as $\mathrm{SNR}=0.655 \cdot \frac{\text { signal intensity }}{\mathrm{SD}_{\text {background }}}$ according to a study by Kaufman et al. [18], while CNR was calculated as $\mathrm{CNR}_{1 / 2}=\frac{\mid \text { signal intensity }{ }_{1}-\text { signal intensity }}{2} \mid . \mathrm{SNR}$ was calculated on the scar tissue $\left(\mathrm{SNR}_{\mathrm{scar}}\right)$, while $\mathrm{CNR}$ was calculated between scar tissue and remote myocardium $\left(\mathrm{CNR}_{\text {scar-rem }}\right)$, and between scar tissue and blood $\left(\mathrm{CNR}_{\text {scar-blood }}\right)$. Timings between contrast injection and acquisition of LGE sequences were also reported.

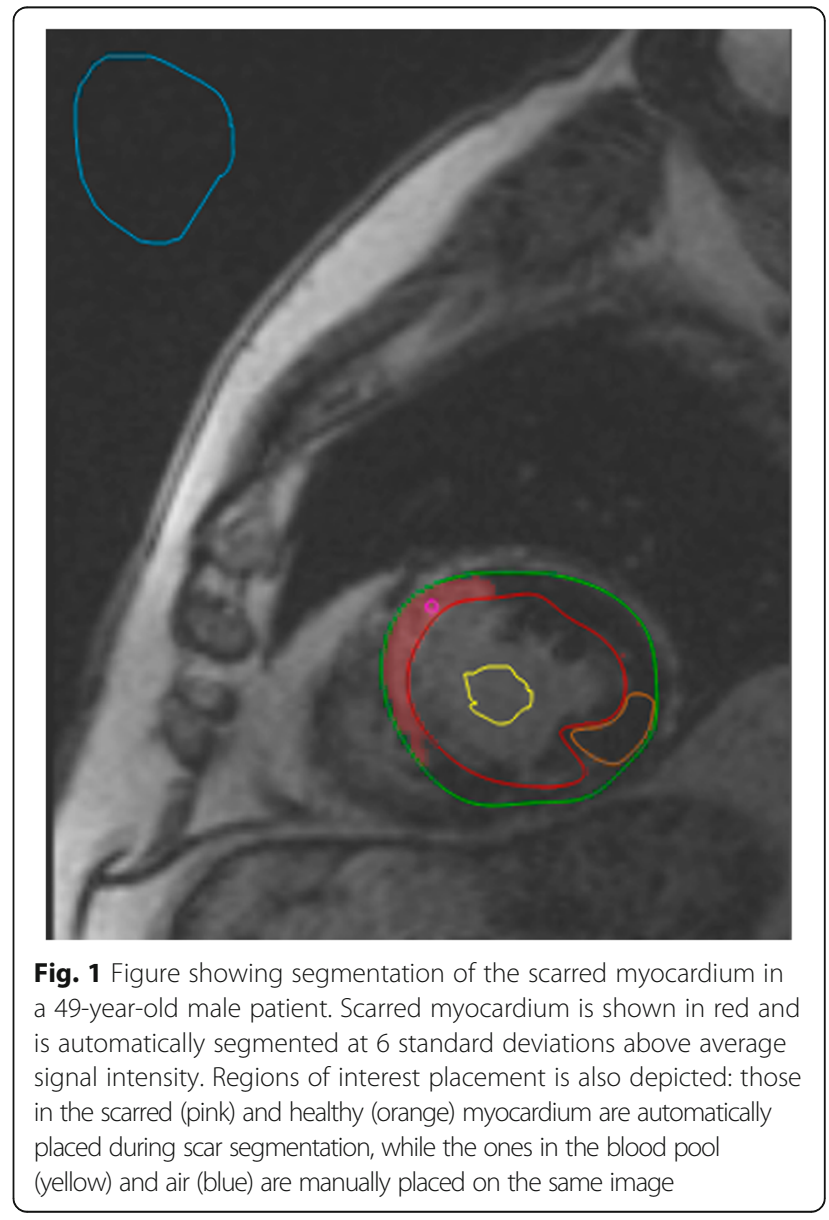


Subjective image quality was also analysed, using a 4point Likert scale, defining score as follows: 0: nondiagnostic; 1: diagnostic exam, sufficient quality; 2: diagnostic exam, good quality; 3: diagnostic exam, excellent quality. The quality definition was based on the visual contrast differences between blood pool signal and LGE.

\section{Statistical analysis}

Data were reported as median and interquartile range (IQR). Differences between groups were appraised with KruskalWallis test for numerical variables, and post hoc tests when a significant difference was appraised by Kruskal-Wallis test, or Fisher $\chi^{2}$ tests for non-numerical variables.

Statistical analysis was performed with MATLAB R2018b (Mathworks, Natick, MA, USA), and $p$ values $\leq$ 0.05 were considered statistically significant.

\section{Results}

\section{Study population}

Out of 124 patients who had undergone contrastenhanced CMR at our institution, with gadobutrol as GBCA, 79 were included. The flowchart of exclusion is shown in Fig. 2. Out of the 79 included patients, 22 belonged to the group being administered $0.10 \mathrm{mmol} / \mathrm{kg}$ of gadobutrol (group A), 26 to the group being administered $0.15 \mathrm{mmol} / \mathrm{kg}$ of gadobutrol (group B) and 31 to the last group, which was administered $0.20 \mathrm{mmol} / \mathrm{kg}$ of gadobutrol (group C). There were no significant differences in either age or sex among the three groups $(p \geq 0.300)$. Group demographics are summarised in Table 1.

The median acquisition time of LGE sequences was 9 min (IQR 8-13 min) for group A, 14 min (IQR 9-17 min) for group B and $17 \mathrm{~min}$ (IQR 14-20 min) for group C. Acquisition time showed a significant difference $(p<$ 0.001) among groups; in particular, it did not differ between group A and group B $(p=0.105)$, but was shorter in group B than group $\mathrm{C}(p<0.018)$, and shorter in group $\mathrm{A}$ than in group $\mathrm{C}(p<0.001)$.

\section{Cardiac morphology and function}

Left ventricular volumetric and functional data are reported in Table 1, along with myocardial scar burden quantified as percentage of scar tissue volume over the whole left ventricular volume. There were no significant differences in volumetric, functional or scar data.

\section{Image quality}

Images of LGE in patients belonging to the three different groups are shown in Fig. 3.

$\mathrm{SNR}_{\text {scar }}$ was 46.4 (IQR 40.3-65.1) in group A, 70.1 (IQR 52.2-111.5) in group $B$, and 72.1 (IQR 59.4-100.0) in group $C$. There was a significant difference in $\mathrm{SNR}_{\text {scar }}$ among groups $(p=0.002)$, in particular $\mathrm{SNR}_{\text {scar }}$ in group A was lower than both that of group B $(p=0.013)$ and group $C(p=0.002)$, while there was no significant difference in $\mathrm{SNR}_{\mathrm{scar}}$ between group $\mathrm{B}$ and group $\mathrm{C}(p=0.884)$.

$\mathrm{CNR}_{\text {scar-rem }}$ was 62.9 (IQR 52.2-87.4) in group A, 96.5 (IQR 73.1-152.8) in group B, and 103.9 (IQR 83.9132.0) in group $C$. There was a significant difference in $\mathrm{CNR}_{\text {scar-rem }}$ among groups $(p<0.001)$, in particular $\mathrm{CNR}_{\text {scar-rem }}$ in group A was significantly lower than both that of group $\mathrm{B}(p=0.008)$ and group $\mathrm{C}(p=0.001)$, while there was no significant difference in $\mathrm{CNR}_{\text {scar-rem }}$ between group B and group C ( $p=0.871)$.

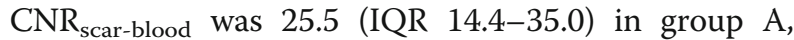
32.7 (IQR 17.9-60.8) in group $B$, and 29.6 (IQR 18.253.5) in group $C$. There were no significant differences in $\mathrm{CNR}_{\text {scar-blood }}$ among groups $(p=0.335)$.

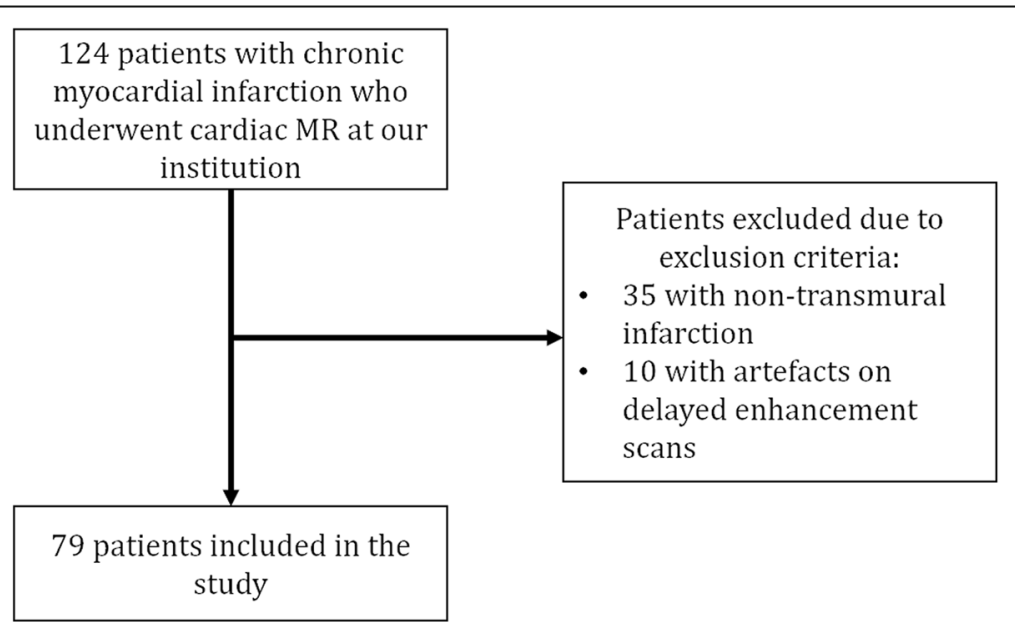

Fig. 2 Study flowchart. Out of 124 initially retrieved patients, 35 were excluded due to their infarction not being transmural and 10 due to artefacts on late gadolinium enhancement scans regardless of the size of their infarction 
Table 1 Demographics, left ventricular function and volume, and scar data from the three study subgroups

\begin{tabular}{|c|c|c|c|c|}
\hline & Group A & Group B & Group C & $p$ \\
\hline Number & 22 & 26 & 31 & - \\
\hline Age (years) & $68(58-71)$ & $62(51-72)$ & $60(51-68)$ & 0.300 \\
\hline Males (\%) & 95 & 92 & 90 & 0.811 \\
\hline LV EDVi $\left(\mathrm{ml} / \mathrm{m}^{2}\right)$ & $94(75-118)$ & $93(73-107)$ & $100(80-126)$ & 0.319 \\
\hline LV ESVi $\left(\mathrm{ml} / \mathrm{m}^{2}\right)$ & $58(31-77)$ & $56(46-74)$ & $63(46-87)$ & 0.472 \\
\hline LV SV (ml) & 66 (46-78) & $69(60-86)$ & $70(62-83)$ & 0.410 \\
\hline LV EF (\%) & $38(28-46)$ & $38(31-47)$ & $37(30-45)$ & 0.800 \\
\hline LV Mi $\left(\mathrm{g} / \mathrm{m}^{2}\right)$ & $89(81-116)$ & 91 (77-114) & $92(77-102)$ & 0.961 \\
\hline LGE (\%) & $32.5(21.7-38.1)$ & $30.9(23.0-42.4)$ & $31.1(25.5-44.0)$ & 0.594 \\
\hline
\end{tabular}

EDVi End-diastolic volume indexed to body surface area, EF Ejection fraction, ESVi End-systolic volume indexed to body surface area, LGE Percentage of scar represented as late gadolinium enhancement over the myocardial mass, Mi Myocardial mass index, SV Stroke volume. Kruskal-Wallis test was used

Box plots of $\mathrm{SNR}_{\text {scar }}, \mathrm{CNR}_{\text {scar-rem }}$, and $\mathrm{CNR}_{\text {scar-blood }}$ across the three groups are depicted in Fig. 4, and data are reported in Table 2.

Concerning subjective image quality, no exams were non-diagnostic (Likert score 0), 7 exams displayed sufficient quality (Likert score 1), 24 exams good quality (Likert score 2), and 48 exams excellent quality (Likert score 3). In group A, 4 exams displayed sufficient quality, 7 good quality, and 11 excellent quality. In group B, 3 exams displayed sufficient quality, 7 good quality, and 16 excellent quality. In group C, 10 exams displayed good quality and 21 excellent quality. There were no significant differences in subjective image quality among groups $(p=0.250)$

\section{Discussion}

The issue of GBCA dose reduction has become crucial in the last few years [19]. Among patients who undergo contrast-enhanced CMR, one of the main groups is represented by patients with chronic myocardial infarction, especially when the infarct is transmural and of greater clinical relevance [20]. In this study, we wished to ascertain whether lower GBCA doses resulted in lower scar image quality, or if there was room for dose reduction while preserving scar visibility. Even lower GBCA doses guarantee diagnostic quality; however, especially given the rise of automatic post-processing methods, it may be important to preserve the highest possible scar discernment to ensure images can be utilised for such purposes. In fact, the quantification of LGE using standard deviations may be influenced by SNR and CNR, as lower SNR and CNR may signify that background noise has a higher impact on intrinsic signal intensity variations, and this may lead to less accurate scar detection, for instance using standard deviation-related systems.

Acquisition time was optimal in all groups, never exceeding $30 \mathrm{~min}$ as recommended by the literature [21]. Moreover, the differences in acquisition timings reflect the recommendations to obtain adequate image contrast according to the dose of contrast agent used [14]. Among our study groups, there were no significant differences in demographics or volumetric or functional left ventricle data and scar percentage over the whole myocardium. This would imply that none of these variables should have influenced the results of our research.

Concerning scar visibility, a lower $\mathrm{SNR}_{\text {scar }}$ (see Table 2) in group A than in both group B and group $C$ could be due to the fact that a $0.10 \mathrm{mmol} / \mathrm{kg}$ GBCA dose was not sufficient to enhance the scarred myocardium in the same way as the two other doses, even though timing

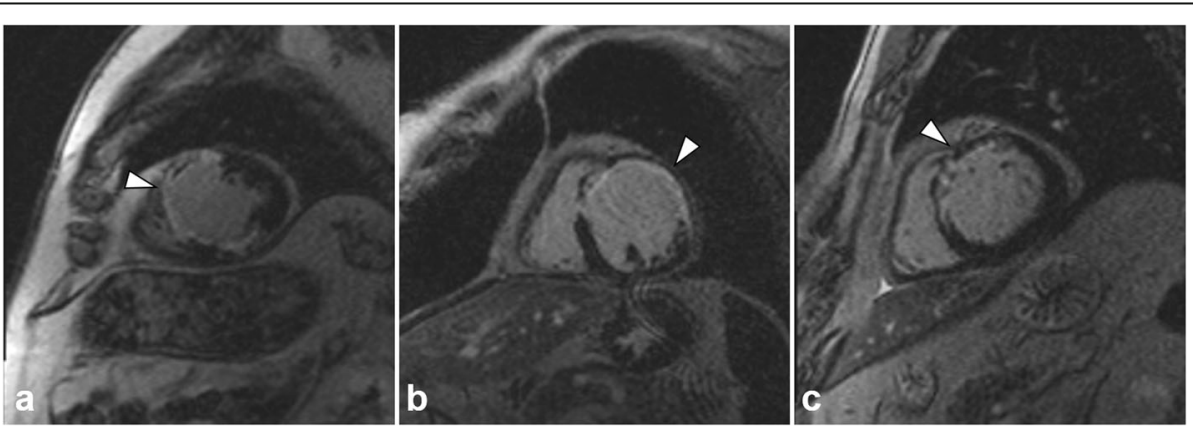

Fig. 3 Inversion recovery sequences for late gadolinium enhancement performed using 0.10 (a), 0.15 (b), or 0.20 (c) $\mathrm{mmol} / \mathrm{kg}$ of gadobutrol, in male patients of 76,54 , and 49 years of age, respectively, matched for percentage infarct size 

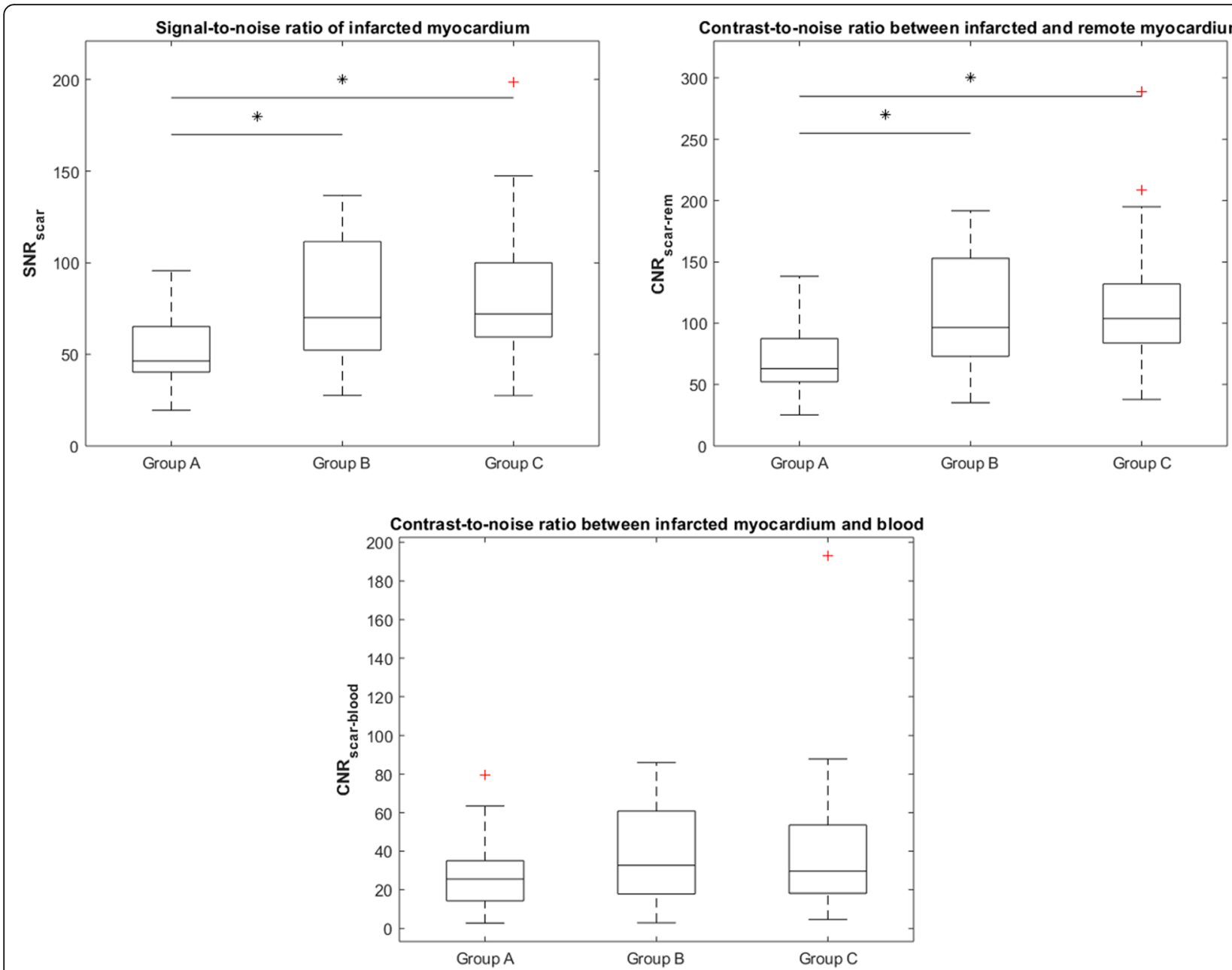

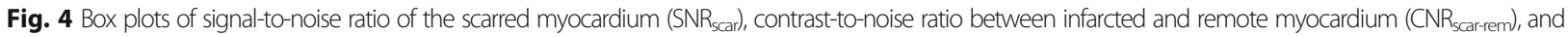
contrast-to-noise ratio between infarcted myocardium and blood ( $\left(\mathrm{CNR}_{\text {scar-blood})}\right.$ in the three groups being administered 0.10 (group A), 0.15 (group B), and 0.20 (group C) mmol/kg of gadobutrol. Significant differences between groups are indicated with an asterisk $\left(^{*}\right)$, and red crosses $(+)$ indicate outliers. In particular,

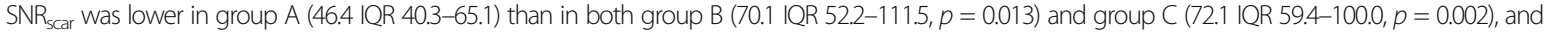
CNRscarrem was lower in group A (62.9 IQR 52.2-87.4) than in both group B (96.5 IQR 73.1-152.8, $p=0.008$ ) and group C (103.9 IQR 83.9-132.0, $p=0.001)$. There were no other significant differences in $\mathrm{SNR}_{\text {scar, }} \mathrm{CNR}_{\text {scar-rem, }}$ or $\mathrm{CNR}_{\text {scar-blood }}(p \geq 0.335)$

Table 2 Image quality and differences among the three groups according to the dose of gadobutrol used for late gadolinium enhancement

\begin{tabular}{llllllll}
\hline & Group A & Group B & Group C & $p$ value (global) & $\begin{array}{l}p \text { value } \\
\text { (A versus B) }\end{array}$ & $\begin{array}{l}p \text { value } \\
(B \text { versus C) }\end{array}$ & $\begin{array}{l}p \text { value } \\
(A \text { versus C) }\end{array}$ \\
\hline SNR $_{\text {inf }}$ & $46.4(40.3-65.1)$ & $70.1(52.2-111.5)$ & $72.1(59.4-100.0)$ & $0.002^{*}$ & $0.013^{*}$ & 0.884 & $0.002^{*}$ \\
CNR $_{\text {scar-rem }}$ & $62.9(52.2-87.4)$ & $96.5(73.1-152.8)$ & $103.9(83.9-132.0)$ & $<0.001^{*}$ & $0.008^{*}$ & 0.871 & $0.001^{*}$ \\
CNR $_{\text {scar-blood }}$ & $25.5(14.4-35.0)$ & $32.7(17.9-60.8)$ & $29.6(18.2-53.5)$ & 0.335 & - & - & - \\
\hline
\end{tabular}

Group A received $0.10 \mathrm{mmol} / \mathrm{kg}$, group B $0.15 \mathrm{mmol} / \mathrm{kg}$, and group C $0.20 \mathrm{mmol} / \mathrm{kg}$ of gadobutrol. CNR scar-blood $_{\text {Contrast-to-noise ratio between myocardial scar }}$ and blood, $C N R_{\text {scar-rem }}$ Contrast-to-noise ratio between scarred and remote healthy myocardium, $S N R_{\text {inf }}$ Signal-to-noise ratio of the myocardial scar. Kruskal-Wallis and Fisher $x^{2}$ tests were used *Indicates statistical significance 
was appropriate for LGE (median 9 min, IQR 8-13 min) [22]. This hypothesis is also supported by a lower $\mathrm{CNR}_{\text {scar-rem }}$ (see Table 2) in group A than in both group $\mathrm{B}$ and group $\mathrm{C}$. $\mathrm{CNR}_{\text {scar-blood }}$ showed no differences (see Table 2) between group A and group B, in accordance with our hypothesis, since both the scarred myocardium and blood are enhanced by the same contrast dose and are still enhanced at the time of LGE acquisition. $\mathrm{SNR}_{\text {scar }}$ was not significantly different between group $\mathrm{B}$ and group $\mathrm{C}$, neither did $\mathrm{CNR}_{\text {scar-rem }}$ and $\mathrm{CNR}_{\text {scar-blood, }}$ suggesting that image quality between the two doses of $0.15 \mathrm{mmol} / \mathrm{kg}$ and $0.20 \mathrm{mmol} / \mathrm{kg}$ of gadobutrol is comparable.

Our results concerning SNR and CNR were not always similar to those obtained by other authors using the same doses of gadobutrol. At $0.10 \mathrm{mmol} / \mathrm{kg}$, our $\mathrm{SNR}_{\text {blood }}$ was lower than that obtained for by De Cobelli et al. [23] using gadobutrol $0.10 \mathrm{mmol} / \mathrm{kg}$ on a group of patients with mixed pathologies exhibiting LGE. Our $\mathrm{CNR}_{\text {scar-rem }}$ was on average slightly lower than theirs but overlapping to a certain degree due to the wide range of distributions; conversely, our $\mathrm{CNR}_{\text {scar-blood was higher. }}$ Their method of calculating SNR was equal to ours except for the lack of the 0.655 adjusting factor which would indeed lower our SNR compared to theirs. Their method of calculating CNR was equal to ours. Concerning $0.15 \mathrm{mmol} / \mathrm{kg}$, both $\mathrm{CNR}_{\text {scar-rem }}$ and $\mathrm{CNR}_{\text {scar-blood }}$ were higher than those obtained by Durmus et al. [24] utilising gadobutrol at $0.15 \mathrm{mmol} / \mathrm{kg}$ with a 15 -min delay to LGE scan. Durmus et al. used the same method for calculating CNR as our study. However, we should consider that our study only included transmural infarctions, while these authors did not exclude patients by scar size. Concerning the comparison of objective image quality parameters, while studies have assessed the differences between different contrast agents at different doses $[25,26]$, to our knowledge none have yet compared different gadobutrol doses.

This study has some limitations, the first being its retrospective design. Results refer to the specific sequence for LGE used at our centre, and to gadobutrol. However, fast inversion-recovery gradient-echo sequences are widely used in clinical practice, and our timings for LGE are aligned to recommendations [21]. On the other hand, gadobutrol is commonly used in CMR [13], it has a double concentration $(1.0 \mathrm{M})$ in comparison with all other vascular/interstitial GBCAs and exhibits an r1-relaxivity relatively higher. However, the double molarity should not impact on LGE findings (obtained after about $10 \mathrm{~min}$ after injection), especially concerning SNR and $\mathrm{CNR}_{\text {scar-rem }}$, as observed by Wildgruber et al. [26], while the clearance of each single GBCA might impact on $\mathrm{CNR}_{\text {scar-blood }}$ Conversely, since the relatively higher relaxivity of gadobutrol may have positively impacted objective image quality of
LGE imaging, as also reported by Schlosser et al. [27], the results obtained for gadobutrol may not be generalizable to GBCAs with a lower relaxivity. Another potential limitation could be posed by the variability of the placement of the regions of interest in the different areas. Nevertheless, the two regions of interest in scarred and healthy myocardium, which were the ones that could carry more issues, were automatically placed by the scar quantification software, and the ones in the air and the blood pool, which were hand-drawn, brought less difficulties. One further limitation, related to the retrospective nature of the study, is represented by the method used for SNR and CNR calculation. In fact, with the only availability of LGE sequences for the assessment of such parameters, the lone viable method for SNR and CNR calculation depended on the use of ROIs placed on the desired structures and background. However, this method has shown to provide the highest variability on SNR in a study by Dietrich et al. [28]. An ideal method for SNR and CNR calculation would perhaps be the one presented by Holtackers et al. [29], who utilised subsequent acquisitions of the same sequence using different inversion times. Nevertheless, we utilised the same sequence for all patients, thus variations in SNR and CNR should be of a systematic nature, thus preserving statistical significance of the observed differences.

In conclusion, results from our study suggest that, while $0.10 \mathrm{mmol} / \mathrm{kg}$ of gadobutrol provides inferior scar image quality of CMI than 0.15 and $0.20 \mathrm{mmol} / \mathrm{kg}$, the last two dosages seem to provide similar LGE. In view of a global trend of standardisation and reduction of GBCA doses, $0.15 \mathrm{mmol} / \mathrm{kg}$ of gadobutrol could be suggested instead of $0.20 \mathrm{mmol} / \mathrm{kg}$, with no hindrance to image quality. Further studies should be conducted to evaluate whether lower GBCA dosages provide a high enough scar quality for clinical evaluations. This would pave the way for further GBCA dose reduction which may impact on image quality, but not on diagnostic utility of CMR examinations.

\section{Abbreviations \\ CMR: Cardiac magnetic resonance; CNR: Contrast-to-noise ratio; \\ $\mathrm{CNR}_{\text {scar-blood: }}$ Contrast-to-noise ratio between scar tissue and blood; $\mathrm{CNR}_{\text {scar-rem: }}$ Contrast-to-noise ratio between scar tissue and remote myocardium; GBCA: Gadolinium-based contrast agents; IQR: Interquartile range; LGE: Late gadolinium enhancement; SNR: Signal-to-noise ratio; $\mathrm{SNR}_{\text {scar: }}$ Signal-to-noise ratio of scar tissue}

\section{Authors' contributions}

FSa, FSe, and MC contributed to the study design. CM, AC, LS, and FSe performed and reviewed image post-processing. CM, MC, and MA performed statistical analysis. All authors participated to manuscript drafting and reviewing. All authors read and approved the final manuscript.

\section{Funding}

This study was partially supported by Ricerca Corrente funding from the Italian Ministry of Health to IRCCS Policlinico San Donato.

Availability of data and materials

All data obtained or analysed during this study are included in this published article. 


\section{Ethics approval and consent to participate}

This study was approved by the Ethics Committee of IRCCS Ospedale San Raffaele (protocol code "Cardioretro", approved on September 14, 2017, and amended on July 18, 2019). Due to the retrospective nature of this study, specific informed consent was waived.

\section{Consent for publication}

As stated in the "Ethics approval and consent to participate" section, consent for publication was also waived due to the retrospective nature of this study.

\section{Competing interests}

Caterina Beatrice Monti, Marina Codari, Andrea Cozzi, Marco Alì, Lorenzo Saggiante, and Francesco Secchi all declare that they have no competing interests. Francesco Sardanelli has received research grants from and is a member of the speakers' bureau and of the advisory group for General Electric, Bayer, and Bracco. In addition, Francesco Sardanelli is the Editor-inChief of European Radiology Experimental; for this reason, he was not involved in any way in the revision/decision process, which was completely managed by the Deputy Editor, Dr. Akos Varga-Szemes (Medical University of South Carolina, Charleston, SC, USA).

\section{Author details}

${ }^{1}$ Department of Biomedical Sciences for Health, Università degli Studi di Milano, Via Mangiagalli 31, 20133 Milano, Italy. ${ }^{2}$ Department of Electronics, Information and Bioengineering, Politecnico di Milano, Via Ponzio 34/5, 20133 Milano, Italy. ${ }^{3}$ Unit of Radiology, IRCCS Policlinico San Donato, Via Morandi 30, 20097 San Donato Milanese, Italy. ${ }^{4}$ Unit of Diagnostic Imaging and Stereotactic Radiosurgery, C.D.I. Centro Diagnostico Italiano S.p.A., Via Saint Bon 20, 20147 Milano, Italy. ${ }^{5}$ Postgraduate School in Radiodiagnostics, Università degli Studi di Milano, Via Festa del Perdono 7, 20122 Milano, Italy. ${ }^{6}$ Department of Biomedical Sciences for Health, Università degli Studi di Milano, Via Morandi 30, 20097 San Donato Milanese, Italy.

Received: 7 November 2019 Accepted: 21 February 2020 Published online: 03 April 2020

\section{References}

1. Townsend N, Wilson L, Bhatnagar P, Wickramasinghe K, Rayner M, Nichols M (2016) Cardiovascular disease in Europe: epidemiological update 2016. Eur Heart J 37:3232-3245. https://doi.org/10.1093/eurheartj/ehw334

2. Antman E, Bassand J-P, Klein W et al (2000) Myocardial infarction redefined-a consensus document of The Joint European Society of Cardiology/American College of Cardiology committee for the redefinition of myocardial infarction. J Am Coll Cardiol 36:959-969. https://doi.org/10. 1016/S0735-1097(00)00804-4

3. Kinno M, Nagpal P, Horgan S, Waller AH (2017) Comparison of echocardiography, cardiac magnetic resonance, and computed tomographic imaging for the evaluation of left ventricular myocardial function: Part 1 (global assessment). Curr Cardiol Rep 19:9. https://doi.org/ 10.1007/s11886-017-0815-4

4. Rajiah P, Desai MY, Kwon D, Flamm SD (2013) MR imaging of myocardial infarction. Radiographics 33:1383-1412. https://doi.org/10.1148/rg 335125722

5. Muscogiuri G, Rehwald WG, Schoepf UJ et al (2017) T(Rho) and magnetization transfer and INvErsion recovery (TRAMINER)-prepared imaging: A novel contrast-enhanced flow-independent dark-blood technique for the evaluation of myocardial late gadolinium enhancement in patients with myocardial infarction. J Magn Reson Imaging 45:1429-1437. https://doi.org/10.1002/jmri.25498

6. Reimer KA, Jennings RB (1979) The "wavefront phenomenon" of myocardial ischemic cell death. II. Transmural progression of necrosis within the framework of ischemic bed size (myocardium at risk) and collateral flow. Lab Invest 40:633-644

7. Kaandorp TAM, Lamb HJ, Viergever EP et al (2007) Scar tissue on contrastenhanced MRI predicts left ventricular remodelling after acute infarction. Heart 93:375-376. https://doi.org/10.1136/hrt.2006.097675

8. Yoneyama K, Venkatesh BA, Bluemke DA, McClelland RL, Lima JAC (2017) Cardiovascular magnetic resonance in an adult human population: serial observations from the multi-ethnic study of atherosclerosis. J Cardiovasc Magn Reson 19:52. https://doi.org/10.1186/s12968-017-0367-1
9. Flett AS, Hayward MP, Ashworth MT et al (2010) Equilibrium contrast cardiovascular magnetic resonance for the measurement of diffuse myocardial fibrosis: Preliminary validation in humans. Circulation 122:138144. https://doi.org/10.1161/CIRCULATIONAHA.109.930636

10. Thomsen HS, Morcos SK, Almén T et al (2013) Nephrogenic systemic fibrosis and gadolinium-based contrast media: Updated ESUR Contrast Medium Safety Committee guidelines. Eur Radiol 23:307-318. https://doi.org/10. 1007/s00330-012-2597-9

11. Kanda T, Fukusato T, Matsuda M et al (2015) Gadolinium-based contrast agent accumulates in the brain even in subjects without severe renal dysfunction: evaluation of autopsy brain specimens with inductively coupled plasma mass spectroscopy. Radiology 276:228-232. https://doi.org/ 10.1148/radiol.2015142690

12. Rossi Espagnet MC, Bernardi B, Pasquini L, Figà-Talamanca L, Tomà $P$, Napolitano A (2017) Signal intensity at unenhanced T1-weighted magnetic resonance in the globus pallidus and dentate nucleus after serial administrations of a macrocyclic gadolinium-based contrast agent in children. Pediatr Radiol 47:1345-1352. https://doi.org/10.1007/s00247-017-3874-1

13. Nacif MS, Arai AA, Lima JA, Bluemke DA (2012) Gadolinium-enhanced cardiovascular magnetic resonance: administered dose in relationship to united states food and drug administration (FDA) guidelines. J Cardiovasc Magn Reson 14:18. https://doi.org/10.1186/1532-429X-14-18

14. Ishida M, Kato S, Sakuma H (2009) Cardiac MRI in ischemic heart disease. Circ J 73:1577-1588

15. Khalid A, Lim E, Chan BT et al (2019) Assessing regional left ventricular thickening dysfunction and dyssynchrony via personalized modeling and 3D wall thickness measurements for acute myocardial infarction. J Magn Reson Imaging 49:1006-1019. https://doi.org/10.1002/jmri.26302

16. Matsumoto H, Matsuda T, Miyamoto K et al (2015) Temporal change of enhancement after gadolinium injection on contrast-enhanced CMR in reperfused acute myocardial infarction. J Cardiol 65:76-81. https://doi.org/ 10.1016/j.jjcc.2014.04.005

17. Flett AS, Hasleton J, Cook C et al (2011) Evaluation of techniques for the quantification of myocardial scar of differing etiology using cardiac magnetic resonance. JACC Cardiovasc Imaging 4:150-156. https://doi.org/ 10.1016/J.JCMG.2010.11.015

18. Kaufman L, Kramer DM, Crooks LE, Ortendahl DA (1989) Measuring signalto-noise ratios in MR imaging. Radiology 173:265-267. https://doi.org/10. 1148/radiology.173.1.2781018

19. Bussi S, Coppo A, Botteron C et al (2018) Differences in gadolinium retention after repeated injections of macrocyclic MR contrast agents to rats. J Magn Reson Imaging 47:746-752. https://doi.org/10.1002/jmri.25822

20. Gotschy A, Niemann M, Kozerke S, Lüscher TF, Manka R (2015) Cardiovascular magnetic resonance for the assessment of coronary artery disease. Int J Cardiol 193:84-92. https://doi.org/10.1016/j.jicard.2014.11.098

21. Vogel-Claussen J, Rochitte CE, Wu KC et al (2006) Delayed enhancement MR imaging: utility in myocardial assessment. Radiographics 26:795-810. https:// doi.org/10.1148/rg.263055047

22. Kellman P, Arai AE (2012) Cardiac imaging techniques for physicians: late enhancement. J Magn Reson Imaging 36:529-542. https://doi.org/10.1002/ jmri.23605

23. De Cobelli F, Esposito A, Perseghin G et al (2012) Intraindividual comparison of gadobutrol and gadopentetate dimeglumine for detection of myocardial late enhancement in cardiac MRI. AJR Am J Roentgenol 198:809-816. https://doi.org/10.2214/AJR.11.7118

24. Durmus T, Schilling R, Doeblin P et al (2011) Gadobutrol for magnetic resonance imaging of chronic myocardial infarction. Invest Radiol 47:183188. https://doi.org/10.1097/RLI.0b013e318236e354

25. Maravilla KR, San-Juan D, Kim SJ et al (2017) Comparison of gadoterate meglumine and gadobutrol in the MRI diagnosis of primary brain tumors: a double-blind randomized controlled intraindividual crossover study (the REMIND Study). AJNR Am J Neuroradiol 38:1681-1688. https://doi.org/10. 3174/ajnr.A5316

26. Wildgruber M, Stadlbauer T, Rasper M et al (2014) Single-dose gadobutrol in comparison with single-dose gadobenate dimeglumine for magnetic resonance imaging of chronic myocardial infarction at 3 T. Invest Radiol 49: 728-734. https://doi.org/10.1097/RLI.0000000000000076

27. Schlosser T, Hunold P, Herborn CU et al (2005) Myocardial infarct: depiction with contrast-enhanced MR imaging — comparison of gadopentetate and gadobenate. Radiology 236:1041-1046. https://doi.org/10.1148/radiol. 2363040220 
28. Dietrich O, Raya JG, Reeder SB, Reiser MF, Schoenberg SO (2007) Measurement of signal-to-noise ratios in MR images: Influence of multichannel coils, parallel imaging, and reconstruction filters. J Magn Reson Imaging 26:375-385. https://doi.org/10.1002/jmri.20969

29. Holtackers RJ, Chiribiri A, Schneider T, Higgins DM, Botnar RM (2017) Darkblood late gadolinium enhancement without additional magnetization preparation. J Cardiovasc Magn Reson 19:64. https://doi.org/10.1186/s12968$017-0372-4$

\section{Publisher's Note}

Springer Nature remains neutral with regard to jurisdictional claims in published maps and institutional affiliations.

\section{Submit your manuscript to a SpringerOpen ${ }^{\circ}$ journal and benefit from:}

- Convenient online submission

- Rigorous peer review

- Open access: articles freely available online

- High visibility within the field

- Retaining the copyright to your article

Submit your next manuscript at $\boldsymbol{\nabla}$ springeropen.com 